\title{
OPEN Excessive energy expenditure due to acute physical restraint disrupts Drosophila motivational feeding response
}

\author{
Jacob Gordon \& Pavel Masek ${ }^{\bowtie}$
}

To study the behavior of Drosophila, it is often necessary to restrain and mount individual flies. This requires removal from food, additional handling, anesthesia, and physical restraint. We find a strong positive correlation between the length of time flies are mounted and their subsequent reflexive feeding response, where one hour of mounting is the approximate motivational equivalent to ten hours of fasting. In an attempt to explain this correlation, we rule out anesthesia side-effects, handling, additional fasting, and desiccation. We use respirometric and metabolic techniques coupled with behavioral video scoring to assess energy expenditure in mounted and free flies. We isolate a specific behavior capable of exerting large amounts of energy in mounted flies and identify it as an attempt to escape from restraint. We present a model where physical restraint leads to elevated activity and subsequent faster nutrient storage depletion among mounted flies. This ultimately further accelerates starvation and thus increases reflexive feeding response. In addition, we show that the consequences of the physical restraint profoundly alter aerobic activity, energy depletion, taste, and feeding behavior, and suggest that careful consideration is given to the time-sensitive nature of these highly significant effects when conducting behavioral, physiological or imaging experiments that require immobilization.

Taste is an ancient chemosensory modality that allows distinguishing between helpful and harmful foods in our environment. Perception of taste is best characterized by the type of motivational response that it elicits ${ }^{1-3}$. These responses are traditionally classified as either appetitive or aversive $e^{4-9}$. Importantly, taste perception is modulated by collective interactions between sensory stimuli, internal satiety mechanisms, and previous experiences ${ }^{10-18}$. As such, when studying gustatory behavior, we must understand taste as a dynamic and multifaceted percept, rather than as a static and universal chemosensory response. An extensive arsenal of genetic techniques, numerous behavioral assays, and a relatively short life cycle makes Drosophila melanogaster a powerful insect model for studying gustatory behavior ${ }^{6,19-24}$.

When the tarsi or proboscis of a fruit fly comes in contact with a stimulus that is perceived as appetitive, proboscis extension may be elicited to initiate feeding ${ }^{1,10,19,25,26}$. This appetitive response can be used as a reliable index for motivational feeding behavior ${ }^{1,10}$. This technique has been used extensively to study numerous intrinsic and extrinsic factors that may drive motivational feeding behavior ${ }^{27-31}$. Intuitively, both the quality and concentration of the tastant affect its palatability, which suggests that the nature of the stimulus itself plays an important role in motivational feeding ${ }^{2,32-36}$. Further, aversive taste conditioning paradigms have demonstrated that associations established in prior experience play a significant role in mediating motivational feeding behavior ${ }^{37,38}$.

Motivational behavior is also mediated by internal signals representative of energy storage. The satiation state of a fly influences the amount of time that it spends foraging, which suggests that feeding behavior is in part driven by nutritional and metabolic states ${ }^{39}$. Furthermore, fasting increases appetitive behavior via changes in neuromodulatory states which are representative of internal nutrient deficits ${ }^{12,20}$, and even gut microbiota play a role in influencing behavioral feeding decisions ${ }^{40}$.

Many protocols that involve studying individual fruit fly behavior share a common set of preparatory techniques. It starts with removal from food, then anesthetization, and ultimately immobilization. The need for immobilization is common across a wide variety of assays that are concerned with real-time, single fly observation. Fly immobilization is necessary to perform live imaging studies ${ }^{41}$, electrophysiological recordings ${ }^{42}$, and 
behavioral studies ${ }^{27}$. Common methods of immobilization include adhesion using myristic acid ${ }^{9}$ nail polish ${ }^{38}$, lodging in a pipette tip ${ }^{1,14}$, or use of a fly collar ${ }^{43}$. Notably, each of these methods rely on physical restraint to immobilize the fly.

When using any paradigm for behavioral measurement, it behooves us to consider the effects of preparatory techniques such as immobilization on the behavior of the subject. This is particularly important when studying behavior, as these effects often have the capacity to confound the measurements of the behavioral phenotype of interest. Given the numerous factors that contribute to motivational feeding behavior, it is reasonable to consider that immobilization could unforeseeably impact feeding behavior in and of itself via unforeseen physiological and behavioral consequences. Here, we report a correlation between the amount of time that flies spend restrained and partially immobilized and the cumulative change in a subsequent motivational feeding behavior over time. We use behavioral, respirometric, and metabolic techniques to elucidate the link between immobilization and increased motivational feeding response in single flies and find a possible mechanism of excessive energy expenditure as the cause of the increase. We propose a model of a stress and anxiety elicited by the restraint, possibly combined with starvation, that is self-maintaining and self-propagating, and is exhibited as a continuous and energetically costly escape-motivated behavior.

\section{Results}

A Proboscis Extension Response (PER) assay offers an excellent measure of motivational feeding behavior, but to run this assay, flies must first be mounted on their backs to free their tarsi and proboscis. Typically, the amount of time allowed for flies to recover once mounted onto a microscopy slide prior to behavioral measurements (tastant presentation) is anywhere between 0.5 and $5 \mathrm{~h}$. We found that there is an unexpected strong correlation between the length of time that flies are mounted prior to measurement, and the rate of PER (Fig. $1 \mathrm{~A}, \mathrm{R}^{2}=0.8487$, $Y=15.09 \mathrm{X}+9.787)$. After five hours, when flies' responses reached $100 \%$, we reduced the concentration of fructose to $10 \mathrm{mM}$, to reduce the probability of PER, and we found that the increase continues with a similar slope as before, reaching $100 \%$ again after 5 additional hours of mounting (Fig. S1A). After fasting periods ranging from 0 to $48 \mathrm{~h}$, we tested PER after $3 \mathrm{~h}$ of mounting (Fig. $1 \mathrm{~B}, \mathrm{R}^{2}=0.9317, \mathrm{Y}=1.975 \mathrm{X}+6.123$ ). This is comparable to PER in flies mounted between 0 and $5 \mathrm{~h}$ after $24 \mathrm{~h}$ of fasting (Fig. 1C). This suggests that to change the rate of PER from approximately 0 to $100 \%$, flies need either $5 \mathrm{~h}$ of mounting, or $48 \mathrm{~h}$ of being fasted. From this, it can be approximated that $1 \mathrm{~h}$ of mounting is comparable to approximately $10 \mathrm{~h}$ of starvation when tested for motivational feeding response.

Assessing starvation, mounting, and anesthesia. Notably, flies that are mounted longer also experienced a longer period of food deprivation prior to PER measurement (from 24 to $29 \mathrm{~h}$ in Fig. 1A). To rule out the possibility that the correlation might be due to additional fasting, we staggered measurements such that all flies were measured after being mounted for $3 \mathrm{~h}$, but were fasted between 24 and $29 \mathrm{~h}$. The correlation between degree of fasting as an isolated variable and PER probability was far weaker, and the regression slope was much smaller (Fig. 1D, $\mathrm{R}^{2}=0.1312, \mathrm{Y}=4.025 \mathrm{X}-51.61$ ). Furthermore, we found that flies mounted for $5 \mathrm{~h}$ show significantly higher rates of PER when compared to flies mounted for $0.5 \mathrm{~h}$ when both groups are measured after $29 \mathrm{~h}$ of fasting (Fig. 1E). This supports the notion that the correlation depicted in Fig. 1A is caused by factors other than fasting alone.

To mount flies, we must use an adhesive. It is possible that chemical or physical effects of adhesive administration may lead to a period of behavioral recovery where the rate of PER gradually increases. We use nail polish to mount flies. To mitigate possible effects of fumes, we mounted flies in a stream of fresh air. To completely avoid any aromatic chemicals, we also used a sealing wax and attached flies by melting a small portion of it. Neither the presence of ventilation, nor the use of a different adhesive substance altered the rate of PER measured at $3 \mathrm{~h}$ post mounting (Fig. S1B).

Since flies are anesthetized with carbon dioxide $\left(\mathrm{CO}_{2}\right)$ prior to mounting, it is possible that $\mathrm{CO}_{2}$ exposure, anesthesia and the following recovery might affect behavioral responses, as was shown in other behaviors ${ }^{44}$, and might result in metabolic changes ${ }^{45}$. To rule out side effects of $\mathrm{CO}_{2}$, we mounted flies using cold anesthesia. We built a custom Peltier element cooling surface that allowed us to gently cool flies at a temperature of $10^{\circ} \mathrm{C}$. Flies recover from this anesthesia within several seconds after mounting as opposed to a $\mathrm{CO}_{2}$ recovery of several minutes. Yet, the cold anesthesia did not alter the increase of PER over time; and the correlation and regression slope remained unchanged (Fig. $S 1 C, \mathrm{R}^{2}=0.8436, \mathrm{Y}=4.025 \mathrm{X}-51.61$ ). However, the possibility remained that both modes of anesthetization produced similar recovery effects. To rule this out, we re-anesthetized flies with $\mathrm{CO}_{2}$ after $0.5,2$, or $4 \mathrm{~h}$ of being mounted, and measured PER $1 \mathrm{~h}$ later. If anesthesia recovery was responsible for the correlation, these flies would exhibit a rate of PER concordant with flies that had been mounted for $1 \mathrm{~h}$ in Fig. 1B. However, in establishing the length of time mounted as an isolated variable, we still found a strong correlation with the rate of PER (Fig. S1D, $\mathrm{R}^{2}=0.4977$ ).

Metabolic measurements. It has been demonstrated that increased hunger is reflective of internal energy storage depletion ${ }^{39}$. Since flies that were mounted for longer exhibited increased motivational feeding behavior, we reasoned that this difference in satiety state should also be reflected in the levels of energy storage. Indeed, flies that were mounted for $5 \mathrm{~h}$ demonstrated significant glycogen depletion compared to flies that were mounted for only $1 \mathrm{~h}$ (Fig. $2 \mathrm{~A}, \mathrm{p}<0.01$ ). Further, flies that were mounted for $5 \mathrm{~h}$ had significantly lower glycogen levels than flies that were free walking for $5 \mathrm{~h}$ after anesthesia (Fig. $2 \mathrm{~A}, \mathrm{p}<0.05$ ). These data show that glycogen is depleted significantly faster among mounted flies when compared to free walking flies, suggesting that there is an additional factor among mounted flies that leads to faster energy depletion. 
A

\section{Total time fasted [hours]}
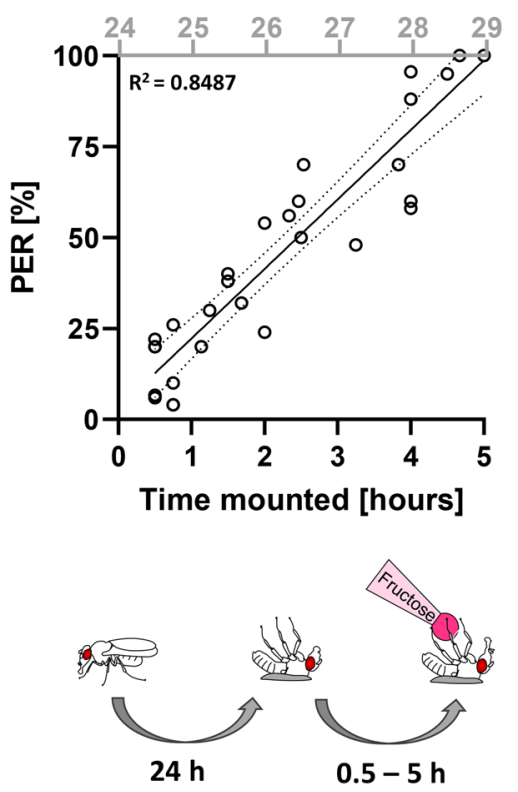

D
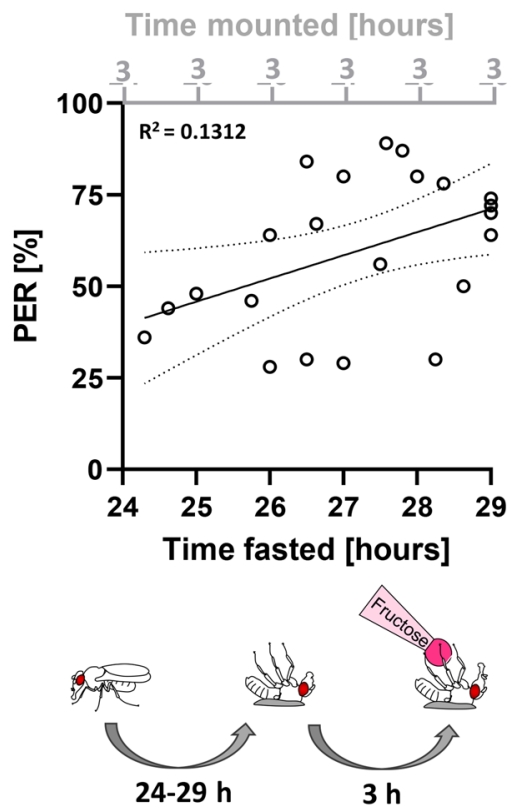

B
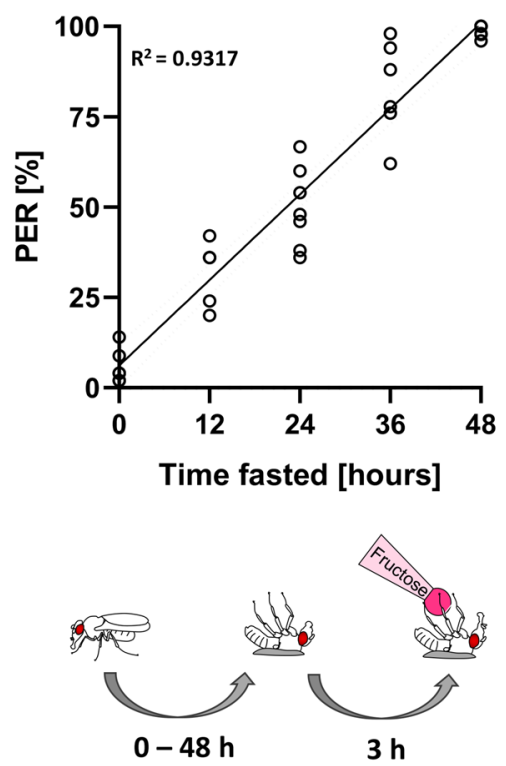

$\mathbf{E}$
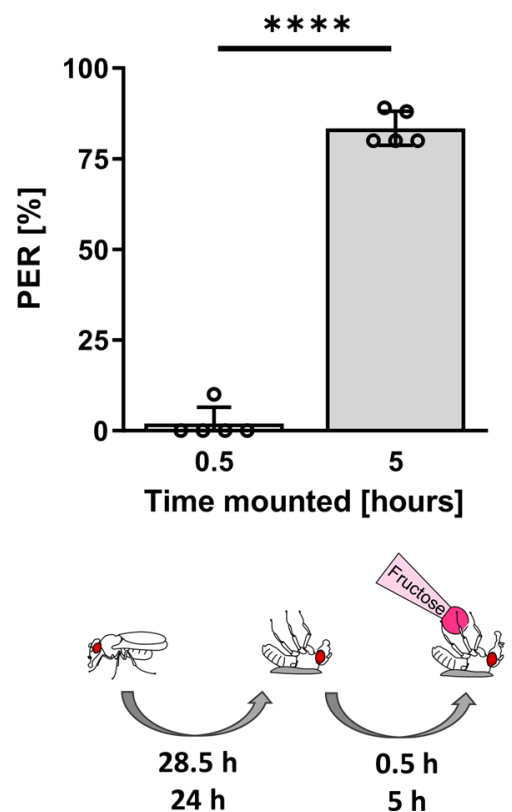

C

Time fasted [hours]

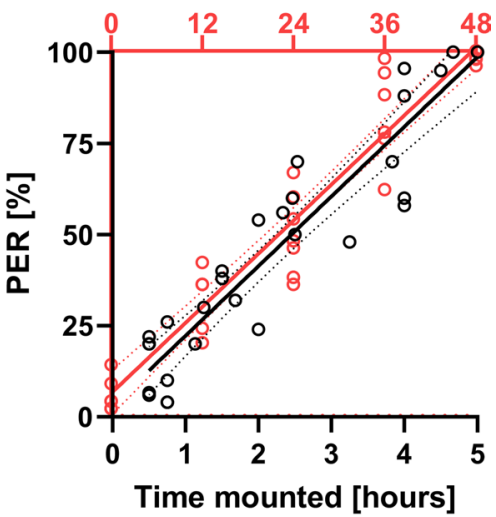

Figure 1. Flies mounted for longer time exhibit heightened motivational feeding response. (A) Average PER to $100 \mathrm{mM}$ fructose of flies mounted between 0.5 and $5 \mathrm{~h}$ prior to testing $(\mathrm{n}=29, \mathrm{~N}=290)$ [simple linear regression]. (B) Average PER to $100 \mathrm{mM}$ fructose among 0-48 h fasted flies after they were mounted for $3 \mathrm{~h}$ prior to testing $(n=30, N=300)$. (C) Overlay of data from 1B with 1A shows an almost perfect overlap with nearly identical slope. (D) Average PER to $100 \mathrm{mM}$ fructose among 24-29 h fasted flies that were all mounted for $3 \mathrm{~h}$ prior to testing $(\mathrm{n}=23, \mathrm{~N}=230)$. (E) Average PER to $100 \mathrm{mM}$ fructose among flies fasted for $28.5 \mathrm{or} 24 \mathrm{~h}$, then mounted for either 0.5 or $5 \mathrm{~h}$ prior to measurement, respectively, with a total fasting time of $29 \mathrm{~h}$ in both groups $\left(n=5, N=50\right.$ per condition ${ }^{* * *} P<0.0001$, t-test).

We also compared glucose levels between flies that were mounted for $1 \mathrm{~h}$ with flies mounted for $5 \mathrm{~h}$, the latter of which had significantly lower glucose levels (Fig. $2 \mathrm{~B}, \mathrm{p}<0.05$ ). On the other hand, free walking flies that 
A

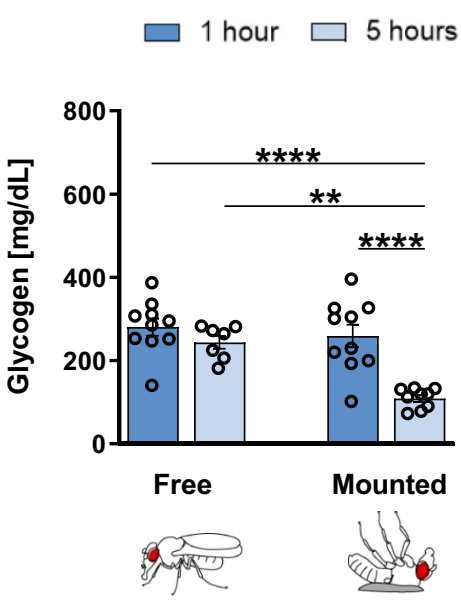

B

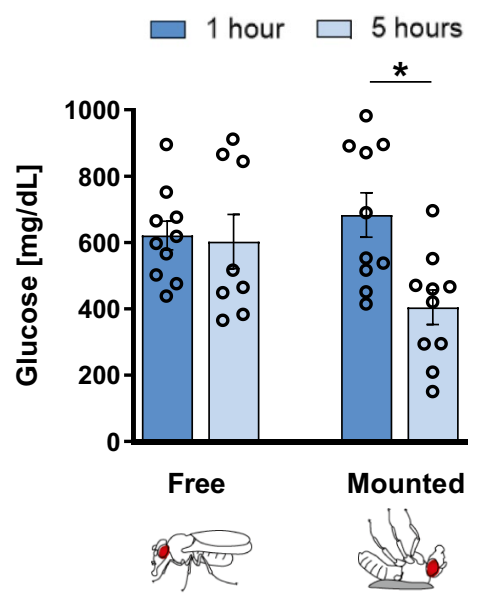

C

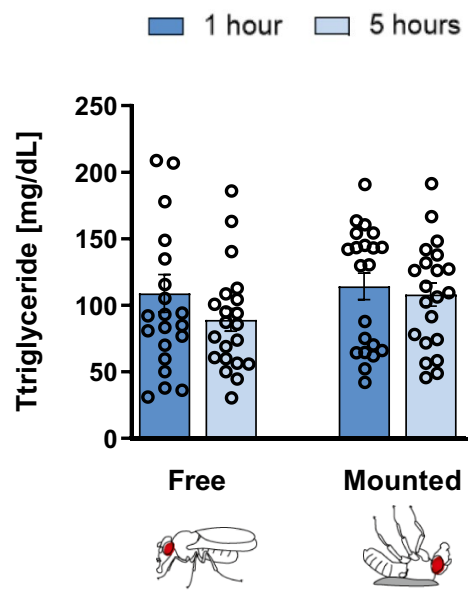

Figure 2. Flies mounted for longer experience depletion of glucose and glycogen storages. (A) Average glycogen $(\mathrm{mg} / \mathrm{dL})$ for free walking flies after $1 \mathrm{~h}(\mathrm{n}=10, \mathrm{~N}=50)$ and $5 \mathrm{~h}(\mathrm{n}=8, \mathrm{~N}=40)$ since anesthetization, and for mounted flies after $1 \mathrm{~h}(\mathrm{n}=10, \mathrm{~N}=50)$ and $5 \mathrm{~h}(\mathrm{n}=10, \mathrm{~N}=50)$ since mounting. (B) Average glucose $(\mathrm{mg} /$ $\mathrm{dL})$ for free walking flies after $1 \mathrm{~h}(\mathrm{n}=10, \mathrm{~N}=50)$ and $5 \mathrm{~h}(\mathrm{n}=8, \mathrm{~N}=40)$ since anesthetization, and for mounted flies after $1 \mathrm{~h}(\mathrm{n}=10, \mathrm{~N}=50)$ and $5 \mathrm{~h}(\mathrm{n}=10, \mathrm{~N}=50)$ since mounting. (C) Average triglyceride $(\mathrm{mg} / \mathrm{dL})$ for free walking flies after $1 \mathrm{~h}(\mathrm{n}=21, \mathrm{~N}=105)$ and $5 \mathrm{~h}(\mathrm{n}=21, \mathrm{~N}=105)$ since anesthetization, and for mounted flies after $1 \mathrm{~h}(\mathrm{n}=21, \mathrm{~N}=105)$ and $5 \mathrm{~h}(\mathrm{n}=21, \mathrm{~N}=105)$ since mounting $\left[{ }^{*} P<0.05,{ }^{* *} P<0.01,{ }^{* * * *} P<0.0001\right.$, ordinary oneway ANOVAs followed by Tukey's multiple comparisons tests].

were measured at 1 and $5 \mathrm{~h}$ after anesthesia administration showed no significant differences with respect to glucose levels (Fig. 2B). This suggests that mounted flies deplete their glucose at a faster rate than free walking flies, albeit the difference in glucose levels between free walking flies and mounted flies at $5 \mathrm{~h}$ was not significant. This is probably because hemolymph glucose is being continuously replenished from glycogen. As hemolymph glucose needs to be kept within a normal range of values, glycogen is likely converted to glucose when energy demand increases.

We saw no significant differences in triglyceride levels among flies mounted for $5 \mathrm{~h}$, flies mounted for $1 \mathrm{~h}$, or free flies (Fig. 2C, p < 0.05), indicating that the increase in the rate of PER among mounted flies is dependent on glycogen but not triglyceride storage depletion.

Activity and respirometry. Free walking flies tend to spend most of their time inactive and thus consume relatively little energy compared to, for example, flying flies ${ }^{46-49}$. We video-recorded free walking flies placed in a small chamber and flies mounted on a slide, and quantified their activity at midday (Fig. 3A, see Methods), and found that mounted flies spend significantly more time being active (Fig. $3 \mathrm{~B}, \mathrm{p}<0.05$ ). To assess the possible confounds of circadian rhythm on activity, we measured flies in the morning and observed the same (lack of) activity in free flies, but lower in mounted flies compared to midday, however, at both times, mounted flies spent significantly more time active compared to free flies (Fig. S2A, p < 0.0001). Starvation-induced hyperactivity has been previously shown in free-walking flies ${ }^{50}$. To assess whether the activity increase in mounted flies is also fasting dependent, we measured the activity of mounted fed flies and flies fasted for 1 or 2 days at $1 \mathrm{~h}$ after being mounted. We found no significant difference in activity as a function of starvation (Fig. S2B).

Next, we used an open-flow respirometer to measure $\mathrm{CO}_{2}$ production in groups of ten flies that were either free walking or mounted (Fig. 3C). Flies that were mounted produced a significantly higher amount of $\mathrm{CO}_{2}$ per second compared to free walking flies (Fig. 3D and Fig. S2, p <0.05). Besides $\mathrm{CO}_{2}$, FlyNap, with an active ingredient of triethylamine, is another anesthetic commonly used to anesthetize Drosophila ${ }^{51}$. Unlike $\mathrm{CO}_{2}$ or cold anesthesia, administration of triethylamine seizes all major motor activity in flies, but maintains normal respiratory function ${ }^{52}$. Respirometric measurements of flies anaesthetized using triethylamine provide a baseline rate of $\mathrm{CO}_{2}$ production and metabolism of a fly without any major movement. We believe that subtracting this baseline measurement from both free walking and mounted flies is an even better representation of their difference in $\mathrm{CO}_{2}$ production (Fig. 3E, $\mathrm{p}<0.0001$ ). These findings suggest that the higher activity of mounted flies results in increased metabolic rate and thus in higher $\mathrm{CO}_{2}$ production. To better link the mounted fly activity and $\mathrm{CO}_{2}$ production, we scored the activity of an individual fly mounted in the respirometer while simultaneously monitoring its levels and $\mathrm{CO}_{2}$ production (Fig. $3 \mathrm{C}$ ). We found that the bouts of activity in mounted flies tends to correspond with increases in $\mathrm{CO}_{2}$ production and that the overall production of $\mathrm{CO}_{2}$ during bouts of activity is significantly higher compared to bouts of inactivity (Fig. 3F, G, $p<0.0001$ ). This supports the notion that higher activity demands more energy which is manifested in greater $\mathrm{CO}_{2}$ production.

Escape-related behavior. The activity of mounted flies, as previously compared to the activity of free walking flies, was scored by observing the presence or absence of leg movements. Closer examination revealed 
A
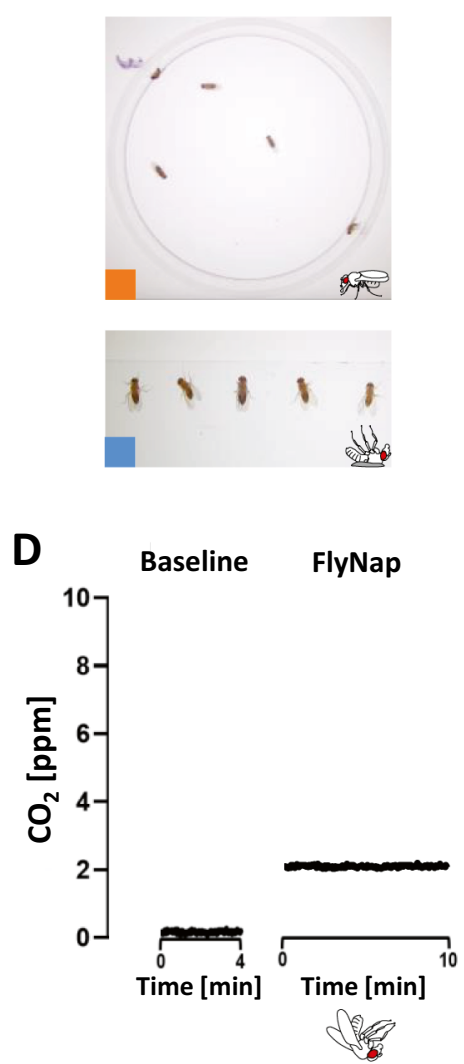

$\mathbf{F}$

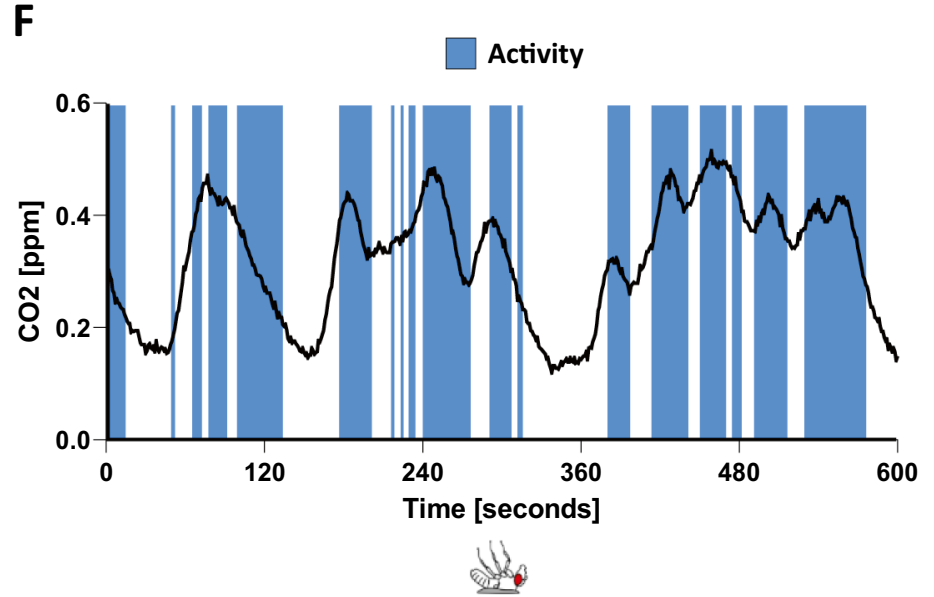

B

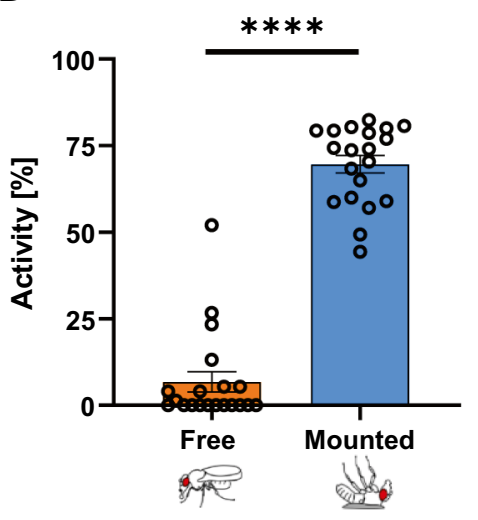

Free

Mounted
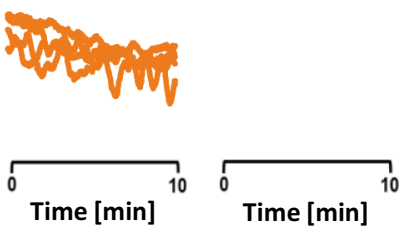

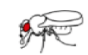

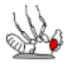

C

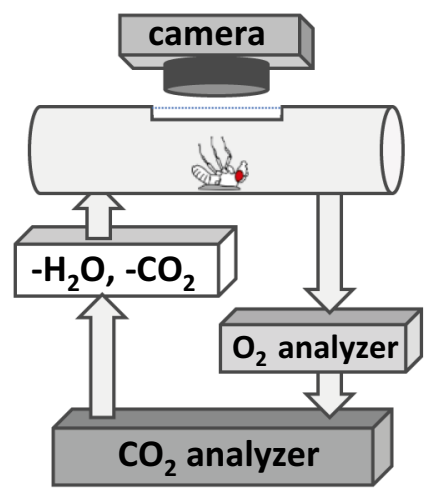

E

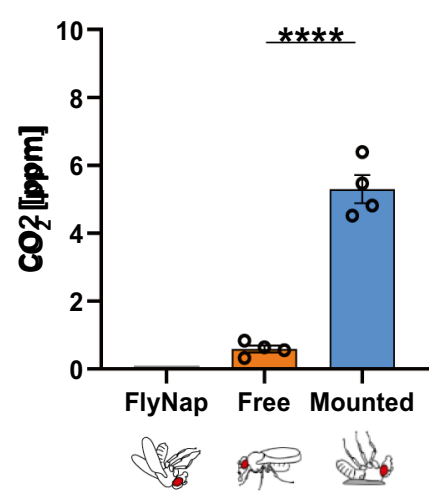

G

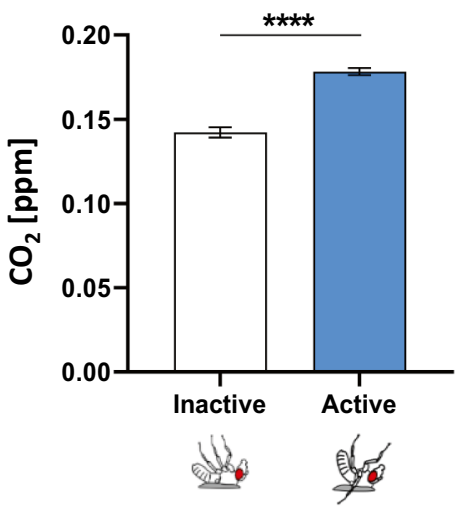

Figure 3. Mounted flies aerobically exert themselves more than free walking flies. (A) The activity of free walking and mounted flies were manually scored over $5 \mathrm{~min}$. (B) Fraction of time spent active for free and mounted ( $n=20$, per condition) flies over $5 \mathrm{~min}$, measured midday $(\mathbf{C})$ Open-flow respirometry setup with live video recording. (D) Average $\mathrm{CO}_{2}$ production over a 10-min interval in free walking $(\mathrm{n}=4, \mathrm{~N}=40)$ and mounted flies $(n=4, N=40)$, compared to machine baseline $(n=5)$, and FlyNap anesthetized flies $(n=4, N=40)$. (E) Average $\mathrm{CO}_{2}$ production in free walking $(\mathrm{n}=4, \mathrm{~N}=40)$ and mounted flies $(\mathrm{n}=4, \mathrm{~N}=40)$ normalized to average $\mathrm{CO}_{2}$ production in FlyNap anesthetized flies $(\mathrm{n}=4, \mathrm{~N}=40)(\mathrm{F})$ Sample of data for continuous $\mathrm{CO}_{2}$ production in a single fly with overlayed bars representing activity bouts $(\mathrm{n}=1)$. $(\mathbf{G})$ Average $\mathrm{CO}_{2}$ production in single flies during periods of inactivity and during bouts of activity $(\mathrm{N}=4)$. [ ${ }^{* * *} \mathrm{P}<0.0001$ unpaired $\mathrm{t}$ tests].

that this activity consisted of three unique behaviors which could be separately assessed: leg kicking, leg pushing, and abdomen curling. When observing a mounted fly carefully, these three behaviors are continuously, and often simultaneously present. We used software to measure changes of pixel light intensity in specific regions of interest on high resolution videos which were positioned to selectively detect each of these three behaviors 
(Fig. 4A-C). This qualitative tracking demonstrates that leg kicking, leg pushing, and abdomen curling can each be assessed independently; but that there is noticeable temporal overlap at low and high time resolution (Fig. 4A-C). It is possible that it is the collective effort of these three behaviors that is responsible for the energy expenditure previously shown. To assess whether one or more of these behaviors is responsible for energy storage depletion, we asked whether preventing flies from kicking their legs would reduce the energy expenditure over time. A small piece of tissue paper (Kimwipe) was placed on fly tarsi (Fig. 4D). This prevented leg kicking behavior while maintaining leg pushing and abdomen curling activity (Fig. 4E). Eliminating leg kicking did not alter the correlation between the length of time mounted and the rate of PER (Fig. 4F, $\mathrm{R}^{2}=0.5893, \mathrm{Y}=13.89 \mathrm{X}+8.359$ for control, $\mathrm{R}^{2}=0.5883, \mathrm{Y}=15.25 \mathrm{X}+4.144$ with Kimwipe). This leaves the leg pushing and abdomen curling as the behaviors accountable for energy expenditure in mounted flies. We speculate that these particular behaviors were instances of escape attempts among restrained flies. In fact, flies mounted to microscopy slides by an inexperienced experimenter often push themselves free with what appears to be significant force. We attempted to reproduce these conditions of poor attachment by using partially dried nail polish. The flies that were attached poorly indeed demonstrated a significantly higher likelihood of successfully peeling themselves from the slide and escaping (Fig. 4G, p < 0.05). By escaping and righting themselves, these flies almost instantly stopped moving and remained relatively stationary, much like the free walking flies we recorded in the previous experiments (video not shown). This suggests that the restrained flies, whether successful or not, likely put in a significant effort to escape, which is a parsimonious explanation for frequent pushing on the microscopy slide while restrained. It has been shown that insects are capable of generating enormous amount of force relative to their body weight ${ }^{53,54}$ and even Drosophila is capable using 15 times more energy while flying than when walking ${ }^{22}$. To estimate the amount of force that a fly is capable of using while pushing against the slide they are attached to, we built a balance ('seesaw') apparatus which allows us to measure how much weight a fly can lift in a position that is similar to how they are mounted. Flies were mounted to a bent pin and placed between two cover slips such that their legs were able to push against them upwards (while the fly was mounted facing down). On the other side of the pin, we hung metal weights of increasing heaviness (Fig. 4H). We scored the number of pushes that moved the fly noticeably away from the slides for one minute (downward arrow in Fig. 4H). Without any added weight, the pin was balanced so that when pushing, flies were moving negligibly more than their own body weight. With no additional weight, they pushed with a very high frequency, close to one push per second (Fig. 4I). The frequency decreased significantly with an additional $5 \mathrm{mg}$, and further with $20 \mathrm{mg}$ (Fig. 4I). Our apparatus failed to hold with higher weights, however, considering that the decrease in number of pushes between 20 and $30 \mathrm{mg}$ was minimal, it is likely that flies are capable of pushing even more weight. Even so, $30 \mathrm{mg}$ weight is nearly forty times the fly's body weight (female, $0.776 \mathrm{mg} \mathrm{N}=80$ ). It is thus likely that pushing is the major source of nutrient storage depletion during prolonged periods of restraint.

\section{Discussion}

We described an effect that acute physical restraint in mounted flies has on subsequent measures of motivational feeding behavior and energy storage levels. This study shows that restraint, an unavoidable part of fly mounting, leads to behavioral changes that can interfere with subsequent behavioral and physiological measurements ${ }^{2,38,55}$. We speculate that any behavioral measure that is dependent on level of fasting will be sensitive to this effect. It has been shown that there is an interplay between olfaction and feeding response ${ }^{56}$, that sleep and sleep deprivation is dependent on satiation ${ }^{50,57}$, and that the formation and persistence of memory depends on the level of perceived sweetness ${ }^{5-60}$. As such, we expect that any measurements relevant to these areas of study are susceptible to confounding starvation effects caused by restraint. Even behaviors that do not have a direct link to taste and feeding can be influenced by the excessive energy loss. Flight in flies is very energetically demanding ${ }^{49,61}$ and mounting flies to flight simulators, often for over $12 \mathrm{~h}$ before measurement, could lead to greater energy depletion, a reduction or alteration in the fly's ability and willingness to fly, and a decrease in ability to perform learning or more complex cognitive tasks ${ }^{62,63}$

There are a multitude of mounting techniques used to study behavior in flies, none of which we believe are entirely immune to the effects we discuss. One common technique used to study fly walking and navigation involves mounting a fly to a ball suspended on air $^{64-67}$. For both flight and walking experiments, flies are attached either to a solid platform, similar to the way we mounted flies in this study, or they are mounted to a thin metal hook between their head and thorax ${ }^{62,68,69}$. Even flies that are mounted to a hook attempt to pull on it with their legs and will occasionally remove themselves in a manner that is similar as depicted in Fig. 4G. We can assume that the escape-related behavior likely uses similar force as is described in this study; and are consequently energetically taxing. Many of these mounting techniques where flies are glued by their thorax, head, or both are also widely used for calcium imaging ${ }^{4,68,70,71}$. If the mounting period is not long, the effects of energy depletion due to restraint-induced struggle may not be significant, however for assays where recording for longer than a few minutes is necessary, confounding effects are possible. In some assays, mounting time can be as long as 12-24 $\mathrm{h}^{2,72,73}$, and because we show the effect is constant for a minimum of $10 \mathrm{~h}$, careful attention should be paid to mitigate this nonspecific variable, especially in these assays.

Some mounting techniques, namely placing flies inside a pipette tip, do not require the use of an adhesive ${ }^{1,9}$. Nevertheless, we assume that, if measured and closely observed, the flies would display signs of struggle, and would likely exert extra energy despite their inability to move their limbs and abdomen along visible trajectories. In such assays where behavioral activity cannot be measured, the depletion of energy storage could be used to assess the degree of struggle in different mounting preparations. In preparations where enclosure in a metabolic chamber is possible, $\mathrm{CO}_{2}$ could be measured in real time to estimate the energy expenditure of mounted flies ${ }^{48}$.

It might seem that using assays where flies are not mounted could eliminate effects associated with the fly's attempt to free itself. However even in some assays where the fly is free to move, but is restricted by walls or water 
A

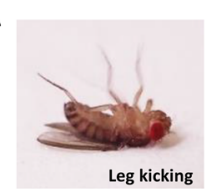

B

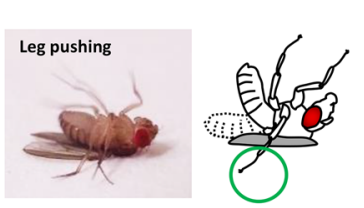

C

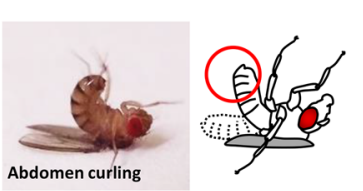

Abdomen curling

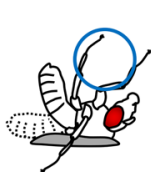

\section{.}

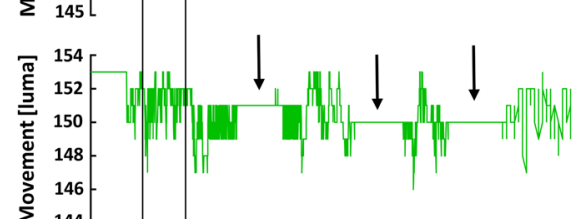

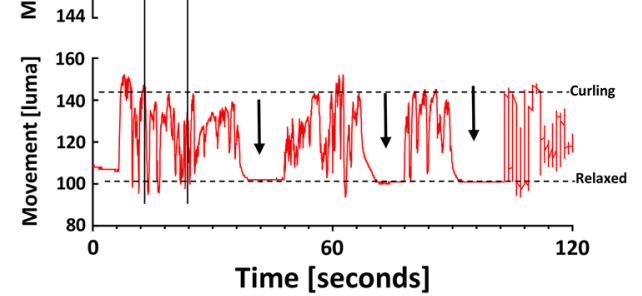

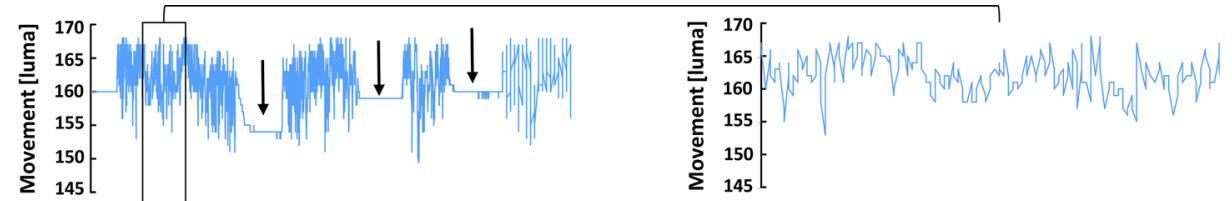

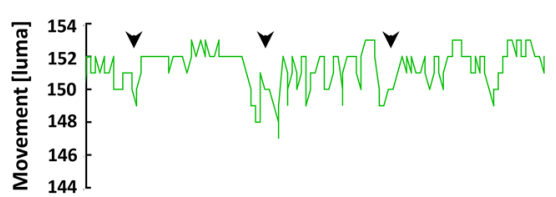

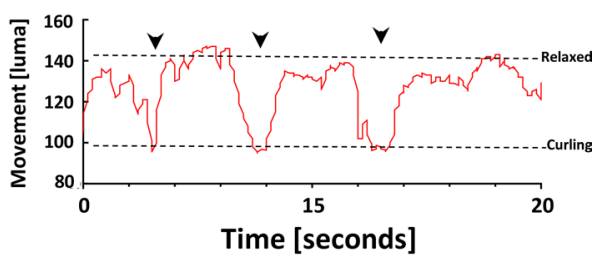

F

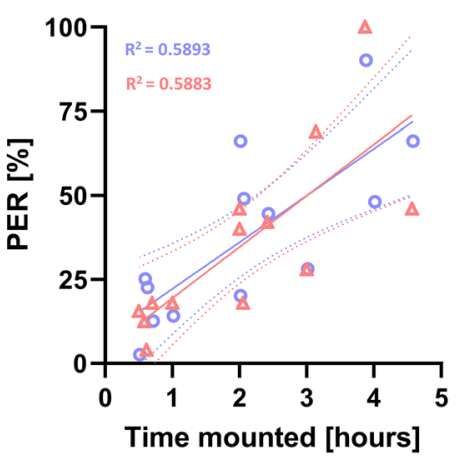

Control

Kimwipe

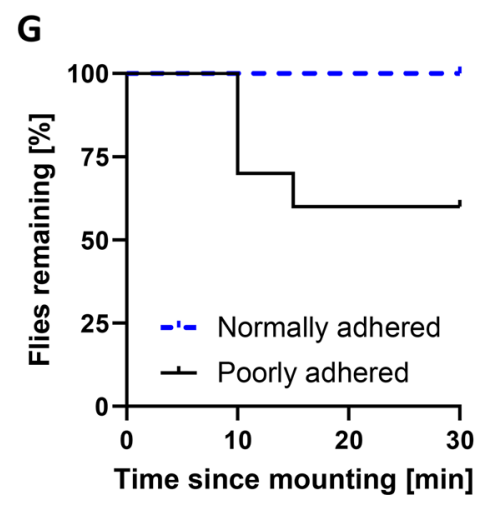

H

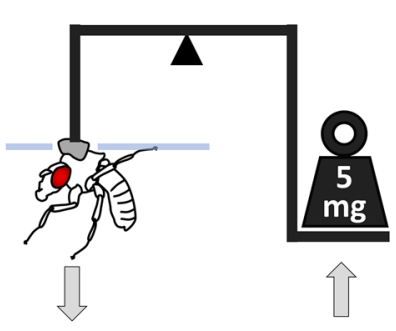

I

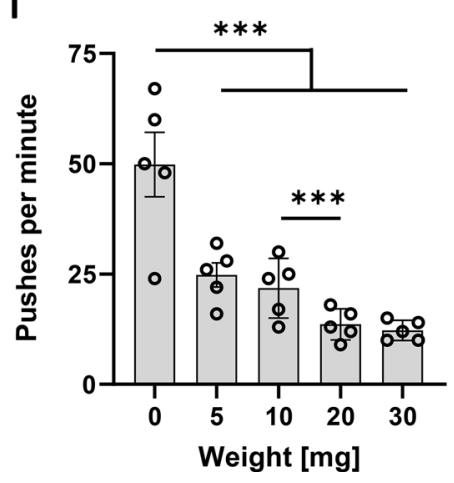

Figure 4. Leg pushing behavior is accountable for increased motivational feeding in restrained flies. (A) Motion detected in region signifying leg kicking behavior in mounted flies, graphically represented by average luminosity in the region over time $(\mathrm{n}=1)$. (B) Motion detected in region signifying leg pushing behavior in mounted flies, graphically represented by average luminosity in the region over time $(n=1)$. (C) Motion detected in region signifying abdomen curling behavior in mounted flies, graphically represented by average luminosity in the region over time $(n=1)$. Arrows show times of inactivity where all behaviors cease. Arrow heads show correlation of an individual leg pushing and abdomen curling. (D) Flies are given a small piece of Kimwipe paper to be held by their tarsi. (E) Average kicking and pushing activity levels for flies with and without pieces of Kimwipe on their tarsi $(n=10, N=100$ per group). (F) Average proboscis extension reflex (PER) in flies with and without pieces of Kimwipe on their tarsi after $0.5-5 \mathrm{~h}$ of being mounted $(\mathrm{n}=13, \mathrm{~N}=130$ per condition). (G) Flies remaining over $30 \mathrm{~min}$ after being normally adhered, and poorly adhered $(\mathrm{n}=10$, $\mathrm{N}=100$ per condition, $\mathrm{p}=0.0289$, survival curve comparison test). $(\mathbf{H})$ Balanced 'seesaw' apparatus which allows individual flies to raise weights during leg pushing behavior (I). Average number of pushes per minute across a range of weights $(\mathrm{N}=6)$. [ ${ }^{* * *} \mathrm{p}<0.001,{ }^{* * * *} \mathrm{p}<0.0001$, unpaired t tests]. 


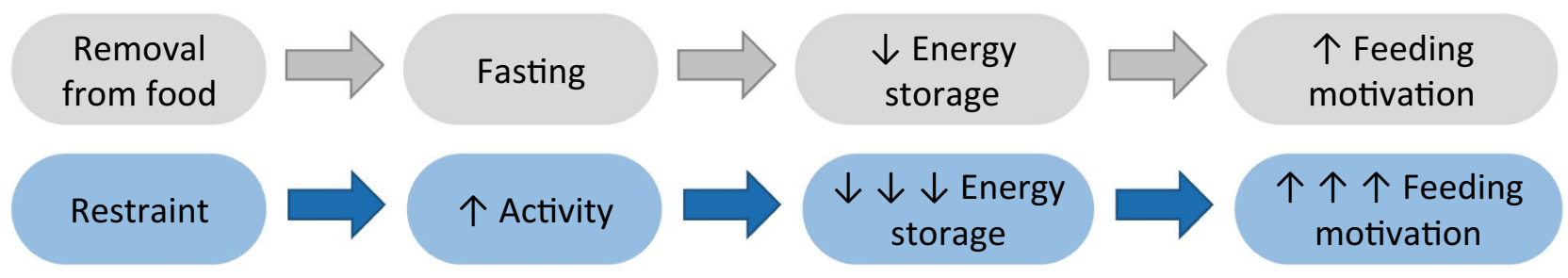

Figure 5. Fasting and restraint additively leads to excessive storage depletion and consequently to elevated feeding motivation. Removal of flies from food leads to fasting that, over time, uses energy storage which can be assessed by measuring a feeding motivation response. Acute physical restraint leads to extended bouts of activity during which we observe elevated levels of energetically demanding behaviors (leg pushing and abdomen curling). This activity depletes internal energy storage far more rapidly than fasting alone, which in turn elevates the internal hunger state and motivational feeding response at a comparatively faster rate. Consequently, the combination of fasting and restraint produces a rise in motivational feeding response over time that is ten times more rapid than from fasting alone.

barriers, there is still an element of entrapment that might motivate attempts to escape ${ }^{47,50,74,75}$. There is no doubt that flies continuously try to escape from restraint or confinement; and anyone who has ever worked with flies has experienced that they often succeed in doing so. In some situations, where escape is impossible, flies exhibit learned helplessness and no longer attempt to escape ${ }^{76,77}$. We have not observed any decrease in escape-related behavior over the course of $10 \mathrm{~h}$, suggesting that this form of restraint does not result in learned helplessness. Flies that are 'trapped' by visible or invisible walls continue to walk for hours, whereas those in relatively free environments tend to spend most of their time standing and grooming ${ }^{47,78,79}$. It would be interesting to measure the extent to which spatially confined, but free walking flies exert energy in their attempts to escape.

The reported effect of $\mathrm{CO}_{2}$ anesthesia on locomotor behavior depends on the length of time flies spend on the $\mathrm{CO}_{2}$ pad and the volumetric flow rate of $\mathrm{CO}_{2}{ }^{44}$. It is possible that we did not see any effect of anesthesia because our mounting technique is fast (approximately $1 \mathrm{~min}$ per slide), and the flow rate of $\mathrm{CO}_{2}$ we use is low. Additionally, we use an in-line water bath to humidify the $\mathrm{CO}_{2}$ and reduce desiccation, which, along with $\mathrm{CO}_{2}$-specific mechanisms and an anoxic effect, causes behavioral deficits after anesthesia ${ }^{44}$. Furthermore, an alternative anesthetic did not alter the correlations (Fig. S1C), supporting the notion that anesthesia, despite its effect on metabolic processes, does not play role in the described effect ${ }^{45}$.

Physical restraint produces an increase of energetically costly physical activity in the form of leg pushing and abdomen curling. This is perhaps a fly's attempt to free itself from restraint, as well as to right itself and regain contact of its tarsi with the ground. Female flies used in our experiments weighed an average of $0.776 \mathrm{mg}$ each, but were capable of moving a $30 \mathrm{mg}$ weight every $6 \mathrm{~s}$. Although this is not as impressive as the strongest reported insect ${ }^{53}$, female flies were still able to lift almost 40 times their body weight repeatedly. We were not able to quantify the energy needed for these lifts, as the weights were moved a very short distance, and leg flexion would further complicate the calculations. However, considering that a standing fly maximally holds its own weight, these data suggest that leg pushing during restraint is highly energetically taxing, even if the behavior is exhibited only about $30 \%$ of the time (Fig. $4 \mathrm{E}$ ).

It is possible that the sustained and elevated activity is caused by stress from being restrained. Stress might parsimoniously explain the motivation underlying the persistent, though often unsuccessful attempts to escape from their restraint. A correlative link between acute restraint and elevated locomotor activity was previously described in mice ${ }^{80,81}$. It was suggested that this relationship is mediated by pathways associated with anxiety behavior ${ }^{82,83}$. Serotonin (5-HT), dopamine and octopamine play an essential role in stress and anxiety in mammals ${ }^{84-86}$ and were all reported to be involved in similar behaviors in flies ${ }^{87-90}$. Therefore, it is possible that neurotransmitters involved in anxiety might mediate the restraint-related behaviors described in this study. Future correlative investigation into the link between restraint, feeding, and energy expenditure in flies may present a novel way to model and study anxiety in invertebrate species.

Based on our data, we propose a model explaining the correlation between the length of time mounted and an increase of motivational feeding response (Fig. 5). When mounted flies attempt to free themselves, they use force that is more than an order of magnitude greater than what is necessary for standing or walking. During extended bouts of activity, the use of this excessive force results in rapid depletion of energy storages such as glycogen. This loss of energy elevates their internal hunger state which manifests as an increase in motivational feeding response.

We show that physical restraint profoundly alters aerobic activity, energy depletion, and feeding behavior. The combination of fasting and restraint produces an increase in motivational feeding response that occurs ten times more rapidly than from fasting alone. This indicates that even a short mounting time can profoundly affect many behavioral, physiological, or imaging experiments requiring immobilization.

\section{Materials and methods}

Experimental animals. Drosophila melanogaster Canton-S flies were grown and maintained on standard food media based on cornmeal/molasses (Jazz mix, Fisher Scientific) and kept in an LD incubator (Powers Scientific; Dros52) at $25^{\circ} \mathrm{C}$ and $60 \%$ humidity on a $12: 12$ light-dark cycle. For all experiments, 3-5 days old, mated 
females were used. All experimental and control groups were measured in parallel at the same Zeitgeber time during midday.

Motivational feeding assay. Flies were fasted for $24 \mathrm{~h}$ prior to preparation for proboscis extension reflex (PER) assay. Flies anesthetized using $\mathrm{CO}_{2}$ were sorted out and size matched females were adhered by their dorsal side of their thorax to a microscopy slide by using nail polish (Cat\#72180, Electron Microscopy Science) as described previously ${ }^{2,38}$. After varying lengths of recovery time, PER was measured as follows: flies were water satiated and were used for experiments only after not responding to water three consecutive times. Flies which continued responding to water after 5-min were excluded from the experiment. Then, $10 \mathrm{mM}$ or $100 \mathrm{mM}$ fructose (CAS\#57-48-7, Sigma Aldrich) was applied briefly (1 s) to the tarsi and the proboscis extension was recorded (only binomial yes/no response was recorded). Each tastant was presented five consecutive times with water used for tarsi washing and satiation (as needed) between each trial. $1 \mathrm{M}$ sucrose (CAS\#57-50-1, Sigma Aldrich) was applied at the end of each session to check for the responsiveness of each fly. Flies that did not respond to sucrose at the end were excluded. An index of PER response was calculated as a percentage of proboscis extensions out of the total number of tastant presentations per fly and per group.

Tests for effects of anesthesia and adhesive. Experiments were performed to test effects of different anesthetics used cold-plate exposure at $10{ }^{\circ} \mathrm{C}$ for $30 \mathrm{~s}$ to anesthetize flies prior to mounting. To test the effects of $\mathrm{CO}_{2}$ anesthesia on PER, 3-5 days old, mated females were fasted for $24 \mathrm{~h}$, then anesthetized using $\mathrm{CO}_{2}$ exposure prior to mounting for $0.5,2.5$, or $4 \mathrm{~h}$. Flies were then anesthetized again using $\mathrm{CO}_{2}$, carefully scraped from the microscopy slide, re-mounted, then tested for PER in response to a $100 \mathrm{mM}$ fructose solution after one additional hour of being mounted.

Otherwise, PER was performed as outlined above. Experiments performed to test the effects of different adhesives used slow airflow using aquarium pump across each microscopy slide as flies were mounted using nail polish, then tested for PER with $100 \mathrm{mM}$ fructose solution after $3 \mathrm{~h}$ of being mounted. Flies were alternatively mounted by applying strips of sealing wax (XICHEN, ASIN-B00Y24YJRU), allowing it to dry, then melting small sections to mount individual flies.

Respirometry. Flies were fasted for $24 \mathrm{~h}$ prior to preparation for respirometry protocols. For mounted flies, flies were anesthetized using $\mathrm{CO}_{2}$ and adhered to a microscopy slide using nail polish as performed in the PER preparatory procedure. Flies were allowed to recover for varying lengths of time and then placed in an open-flow respirometer. Free flies were anesthetized using $\mathrm{CO}_{2}$ and placed in a humidified vial for varying lengths of time. These flies were transferred to a custom-made observation chamber using mouth aspirator and placed inside an open-flow respirometer. After placed inside of the $\mathrm{CO}_{2}$ chamber, flies were allowed to settle for 5 min and only the following $10 \mathrm{~min}$ were used for measurements. $\mathrm{CO}_{2}$ production and video taken through window in the $\mathrm{CO}_{2}$-recording chamber were recorded simultaneously.

Behavioral activity scoring. Individual fly behavior was manually scored per second as either active or inactive. Mounted flies that were moving their legs (kicking) or were pushing against the slide (correlated with extreme abdomen curling), were scored as active. Free walking flies that were walking or running were scored active and those still or grooming were scored as inactive. Tracker software (https://physlets.org/tracker) was used to distinguish three distinct behaviors in mounted flies (leg kicking, leg pushing, abdomen curling) by monitoring average luma in each selected region of interest (ROI) over time as outlined in Fig. 4A-C.

Leg kicking restriction experiment. Flies were fasted for $24 \mathrm{~h}$ and were anesthetized and mounted as previously described. These flies were split into two groups. In one group, each fly was given a torn piece of Kimwipe paper (approximately $2 \times 2 \mathrm{~mm}$ ) to be held by their tarsi; while the control group remained free to kick their legs. Both groups were placed in a humidified chamber and monitored by an observer continuously. Any fly that dropped their Kimwipe paper had it immediately placed back on their tarsi. Any disturbances or removals from humidified chamber were mirrored in the control group. Manual behavioral activity scoring was performed as described above. PER to $100 \mathrm{mM}$ fructose solution was measured in both groups of flies after varying lengths of time mounted, as described previously.

Escape experiment. Flies were fasted for $24 \mathrm{~h}$ and were then split into two groups. One group was adhered with nail polish normally as previously described. The second group was purposefully adhered poorly by allowing each spot of nail polish on the microscopy slide to dry for approximately $5 \mathrm{~s}$ prior to mounting each fly. The number of flies remaining on the microscopy slide for each group was monitored over $30 \mathrm{~min}$.

Metabolic assay fly preparation. Flies were collected and placed on fresh food for $24 \mathrm{~h}$, then starved for $24 \mathrm{~h}$ in food-vials on wet Kimwipe paper. Flies anaesthetizing on $\mathrm{CO}_{2}$ pad and groups of five flies were adhered onto each microscopy slide. The combined weight of each microscopy slide plus five flies was recorded after gluing. Flies were left to recover in a box with wet paper towel for either $1 \mathrm{~h}$ or $5 \mathrm{~h}$. After mounted time elapsed, flies were given water until satiation and then were weighed again. This mostly led to equal weight measured directly after gluing suggesting that weight lost during gluing was mostly due to desiccation. Flies were then anaesthetized and scraped off the microscopy slide using a razor blade and frozen in $1.5 \mathrm{~mL}$ Eppendorf tubes.

Free-walking control flies were anaesthetizing on $\mathrm{CO}_{2}$ pad and groups of five flies were placed in vials on wet Kimwipe paper for either $1 \mathrm{~h}$ or $5 \mathrm{~h}$. Flies were then anaesthetized and frozen in $1.5 \mathrm{~mL}$ Eppendorf tubes. 
Glucose, glycogen, and triglyceride analysis. Eppendorf tubes with five flies were kept on ice until the flies were homogenized in $100 \mu \mathrm{L}$ of phosphate-buffered saline (PBS). The samples were centrifuged at $13,000 \mathrm{rpm}$ for $10 \mathrm{~min}$ at $4{ }^{\circ} \mathrm{C}$ to remove body parts and flakes of nail polish. The supernatant and top fatty layer of each sample was transferred to a new $1.5 \mathrm{~mL}$ Eppendorf tube. Fat was resuspended in the supernatant by vortexing, creating a crude suspension of cell lysate.

For glucose analysis, $2 \mu \mathrm{L}$ of crude lysate for each was added to $98 \mu \mathrm{L}$ of Infinity Glucose reagent (Cat\#TR15421, Thermo Scientific) in a 96-well plate. The plate was incubated at $37^{\circ} \mathrm{C}$ for $5 \mathrm{~min}$, then read at $340 \mathrm{~nm}$. Readings were compared to standard solutions of $0,25,50,100,250,500,1000 \mathrm{mg} / \mathrm{dL}$ glucose.

Glycogen assays were run with the same samples simultaneously with the glucose assay, on the same 96-well plate. $1 \mu \mathrm{L}$ of amyloglucosidase (Sigma A1602-25MG) was added per mL of Infinity Glucose reagent in wells intended for glycogen analysis. $2 \mu \mathrm{L}$ of each lysate sample was added to $198 \mu \mathrm{L}$ of amyloglucosidase and Infinity glucose (or $2 \mu \mathrm{L}$ of lysate to $98 \mu \mathrm{L}$ of plain Infinity glucose reagent) mixture. The plate was incubated for $5 \mathrm{~h}$ at $37^{\circ} \mathrm{C}$ and read $340 \mathrm{~nm}$. Readings were compared to standard solutions of $0,25,50,100,250,500,1000 \mathrm{mg} / \mathrm{dL}$ glucose.

For triglyceride analysis, Eppendorf tubes with five flies were kept on ice until the flies homogenized in 200 $\mu \mathrm{L}$ PBS $+0.1 \%$ Tween. Homogenized samples were heated at $65^{\circ} \mathrm{C}$ for $5 \mathrm{~min}$ to inactivate lipases. $2 \mu \mathrm{L}$ of crude lysate and add to $198 \mu \mathrm{L}$ of Infinity TAG reagent (Cat\#TR22421, Thermo Scientific) for each sample in a 96-well plate. Samples were incubated at $37^{\circ} \mathrm{C}$ for $5 \mathrm{~min}$ then read in a plate reader at $\sim 540 \mathrm{~nm}$. Readings were compared to standard solutions of 0, 10, 50, 100, 150, 200, 400, $600 \mathrm{mg} / \mathrm{dL}$ TAGs. Cayman Chemical (Item \#10010509) was used for this assay.

Statistical analysis. Values for all experiments are displayed as mean \pm SEM with individual values showed where possible. Unpaired t-test was used to test for significance from zero. Paired t-test was used to test for significance between two groups. Survival curve comparison was performed using the Mantel-Cox (log-rank) test. One-way ANOVA with tests of multiple comparisons were performed to compare groups in all assays. Statistical analyses and data presentation were performed using Prism software (GraphPad Software 8.0; San Diego, CA, USA).

Reproducibility statement. For all experiments, 'n' denotes the number of independent measures, while ' $\mathrm{N}$ ' represents the total number of experimental animals. All major experiments were repeated on two or more non-consecutive days to assure reproducibility of results (Figs. 1A-D, 2A-C, 3D-G, 4F, Fig. S2B,C).

Received: 27 April 2021; Accepted: 3 December 2021

Published online: 17 December 2021

\section{References}

1. Shiraiwa, T. \& Carlson, J. R. Proboscis extension response (PER) assay in Drosophila. J. Vis. Exp. https://doi.org/10.3791/193 (2007).

2. Masek, P. \& Scott, K. Limited taste discrimination in Drosophila. Proc. Natl. Acad. Sci. USA 107, 14833-14838 (2010).

3. Chia, J. \& Scott, K. Activation of specific mushroom body output neurons inhibits proboscis extension and sucrose consumption. PLoS ONE 15, e0223034 (2020).

4. Marella, S. et al. Imaging taste responses in the fly brain reveals a functional map of taste category and behavior. Neuron 49, 285-295 (2006).

5. Jeong, Y. T. et al. An odorant-binding protein required for suppression of sweet taste by bitter chemicals. Neuron 79, 725-737 (2013).

6. Montell, C. Drosophila sensory receptors-a set of molecular Swiss Army Knives. Genetics 217, 1-34 (2021).

7. Dahanukar, A., Lei, Y.-T., Kwon, J. Y. \& Carlson, J. R. Two Gr genes underlie sugar reception in Drosophila. Neuron 56, 503-516 (2007).

8. Shim, J. et al. The full repertoire of Drosophila gustatory receptors for detecting an aversive compound. Nat. Commun. 6, 8867 (2015).

9. Devineni, A. V., Sun, B., Zhukovskaya, A. \& Axel, R. Acetic acid activates distinct taste pathways in Drosophila to elicit opposing, state-dependent feeding responses. Elife 8, e47677 (2019).

10. Dethier, V. G. The Hungry Fly (Harvard University Press, 1976).

11. Masek, P., Worden, K., Aso, Y., Rubin, G. M. \& Keene, A. C. A dopamine-modulated neural circuit regulating aversive taste memory in Drosophila. Curr. Biol. 25, 1535-1541 (2015).

12. Marella, S., Mann, K. \& Scott, K. Dopaminergic modulation of sucrose acceptance behavior in Drosophila. Neuron 73, 941-950 (2012).

13. Wang, Q.-P. et al. Sucralose promotes food intake through NPY and a neuronal fasting response. Cell Metab. 24, 75-90 (2016).

14. Yang, Z. et al. A post-ingestive amino acid sensor promotes food consumption in Drosophila. Cell Res. 28, 1013-1025 (2018).

15. Albin, S. D. et al. A subset of serotonergic neurons evokes hunger in adult Drosophila. Curr. Biol. 25, 2435-2440 (2015).

16. Chung, B. Y. et al. Drosophila neuropeptide F signaling independently regulates feeding and sleep-wake behavior. Cell Rep. 19, 2441-2450 (2017).

17. Park, J. H., Carvalho, G. B., Murphy, K. R., Ehrlich, M. R. \& Ja, W. W. Sucralose suppresses food intake. Cell Metab. 25, 484-485 (2017).

18. Musso, P.-Y. et al. Closed-loop optogenetic activation of peripheral or central neurons modulates feeding in freely moving Drosophila. Elife 8, e45636 (2019).

19. Ishimoto, H. \& Tanimura, T. Molecular neurophysiology of taste in Drosophila. Cell. Mol. Life Sci. CMLS 61, 10-18 (2004).

20. Pool, A.-H. \& Scott, K. Feeding regulation in Drosophila. Curr. Opin. Neurobiol. 29, 57-63 (2014).

21. Scott, K. Gustatory Processing in Drosophila melanogaster. Annu. Rev. Entomol. 63, 15-30 (2018).

22. Ling, F., Dahanukar, A., Weiss, L. A., Kwon, J. Y. \& Carlson, J. R. The molecular and cellular basis of taste coding in the legs of Drosophila. J. Neurosci. 34, 7148-7164 (2014).

23. Freeman, E. G. \& Dahanukar, A. Molecular neurobiology of Drosophila taste. Curr. Opin. Neurobiol. 34, 140-148 (2015). 
24. Chen, Y.-C.D., Park, S. J., Ja, W. W. \& Dahanukar, A. Using pox-neuro (Poxn) mutants in Drosophila gustation research: A doubleedged sword. Front. Cell. Neurosci. 12, 382 (2018).

25. Youn, H., Kirkhart, C., Chia, J. \& Scott, K. A subset of octopaminergic neurons that promotes feeding initiation in Drosophila melanogaster. PLoS ONE 13, e0198362 (2018).

26. Chyb, S., Dahanukar, A., Wickens, A. \& Carlson, J. R. Drosophila Gr5a encodes a taste receptor tuned to trehalose. Proc. Natl. Acad. Sci. U. S. A. 100 (Suppl 2), 14526-14530 (2003).

27. Amrein, H. \& Thorne, N. Gustatory perception and behavior in Drosophila melanogaster. Curr. Biol. 15, R673-684 (2005).

28. Thorne, N. et al. Taste perception and coding in Drosophila. Curr. Biol. 14, 1065-1079 (2004).

29. Pool, A.-H. et al. Four GABAergic interneurons impose feeding restraint in Drosophila. Neuron 83, 164-177 (2014).

30. Jiao, Y., Moon, S. J., Wang, X., Ren, Q. \& Montell, C. Gr64f is required in combination with other gustatory receptors for sugar detection in Drosophila. Curr. Biol. 18, 1797-1801 (2008).

31. Nakamura, M., Baldwin, D., Hannaford, S., Palka, J. \& Montell, C. Defective proboscis extension response (DPR), a member of the Ig superfamily required for the gustatory response to salt. J. Neurosci. 22, 3463-3472 (2002).

32. Zhang, Y. V., Ni, J. \& Montell, C. The molecular basis for attractive salt-taste coding in Drosophila. Science 340, 1334-1338 (2013).

33. Ganguly, A. et al. A molecular and cellular context-dependent role for Ir76b in detection of amino acid taste. Cell Rep. 18, 737-750 (2017).

34. Ahn, J.-E., Chen, Y. \& Amrein, H. Molecular basis of fatty acid taste in Drosophila. Elife 6, e30115 (2017).

35. Masek, P. \& Keene, A. C. Drosophila fatty acid taste signals through the PLC pathway in sugar-sensing neurons. PLoS Genet. 9, e1003710 (2013)

36. Kim, H. et al. Drosophila Gr64e mediates fatty acid sensing via the phospholipase C pathway. PLoS Genet. 14, e1007229 (2018).

37. Medioni, J. \& Vaysse, G. Suppression of the tarsal reflex by associative conditioning in Drosophila melanogaster: Acquisition and extinction. C. R. Seances Soc. Biol. Fil. (1975).

38. Keene, A. C. \& Masek, P. Optogenetic induction of aversive taste memory. Neuroscience 222, 173-180 (2012).

39. Beckwith, E. J. \& French, A. S. Sleep in Drosophila and its context. Front. Physiol. 10, 1167 (2019).

40. Leitão-Gonçalves, R. et al. Commensal bacteria and essential amino acids control food choice behavior and reproduction. PLoS Biol. 15, e2000862 (2017).

41. Wu, J. S. \& Luo, L. A protocol for dissecting Drosophila melanogaster brains for live imaging or immunostaining. Nat. Protoc. 1, 2110-2115 (2006).

42. Seelig, J. D. et al. Two-photon calcium imaging from head-fixed Drosophila during optomotor walking behavior. Nat. Methods 7 , 535-540 (2010).

43. Makos, M. A., Omiatek, D. M., Ewing, A. G. \& Heien, M. L. Development and characterization of a voltammetric carbon-fiber microelectrode pH sensor. Langmuir 26, 10386-10391 (2010).

44. Bartholomew, N. R., Burdett, J. M., VandenBrooks, J. M., Quinlan, M. C. \& Call, G. B. Impaired climbing and flight behaviour in Drosophila melanogaster following carbon dioxide anaesthesia. Sci. Rep. 5, 15298 (2015).

45. Colinet, H. \& Renault, D. Metabolic effects of CO(2) anaesthesia in Drosophila melanogaster. Biol. Lett. 8, 1050-1054 (2012).

46. Joiner, W. J., Crocker, A., White, B. H. \& Sehgal, A. Sleep in Drosophila is regulated by adult mushroom bodies. Nature 441, 757-760 (2006).

47. Garbe, D. S. et al. Context-specific comparison of sleep acquisition systems in Drosophila. Biol. Open 4, 1558-1568 (2015).

48. Lehmann, F. O. Matching spiracle opening to metabolic need during flight in Drosophila. Science 294, 1926-1929 (2001).

49. Lehmann, F.-O. \& Schützner, P. The respiratory basis of locomotion in Drosophila. J. Insect Physiol. 56, 543-550 (2010).

50. Murakami, K. et al. Translin is required for metabolic regulation of sleep. Curr. Biol. 26, 972-980 (2016).

51. Seiger, M. B. \& Kink, J. F. The effect of anesthesia on the photoresponses of four sympatric species of Drosophila. Behav. Genet. 23, 99-104 (1993).

52. Tuthill, B. F., Searcy, L. A., Yost, R. A. \& Musselman, L. P. Tissue-specific analysis of lipid species in Drosophila during overnutrition by UHPLC-MS/MS and MALDI-MSI. J. Lipid Res. 61, 275-290 (2020).

53. Knell, R. J. \& Simmons, L. W. Mating tactics determine patterns of condition dependence in a dimorphic horned beetle. Proc. Biol. Sci. 277, 2347-2353 (2010).

54. Full, R., Yamauchi, A. \& Jindrich, D. Maximum single leg force production: Cockroaches righting on photoelastic gelatin. J. Exp. Biol. 198, 2441-2452 (1995).

55. Kirkhart, C. \& Scott, K. Gustatory learning and processing in the Drosophila mushroom bodies. J. Neurosci. 35, 5950-5958 (2015).

56. Shiraiwa, T. Multimodal chemosensory integration through the maxillary palp in Drosophila. PLoS ONE 3, e2191 (2008).

57. Seidner, G. et al. Identification of neurons with a privileged role in sleep homeostasis in Drosophila melanogaster. Curr. Biol. 25, 2928-2938 (2015).

58. Huetteroth, W. et al. Sweet taste and nutrient value subdivide rewarding dopaminergic neurons in Drosophila. Curr. Biol. 25, 751-758 (2015).

59. Musso, P.-Y., Lampin-Saint-Amaux, A., Tchenio, P. \& Preat, T. Ingestion of artificial sweeteners leads to caloric frustration memory in Drosophila. Nat. Commun. 8, 1803 (2017).

60. Gruber, F. et al. Suppression of conditioned odor approach by feeding is independent of taste and nutritional value in Drosophila. Curr. Biol. 23, 507-514 (2013).

61. Lehmann, F.-O. \& Heymann, N. Unconventional mechanisms control cyclic respiratory gas release in flying Drosophila. J. Exp. Biol. 208, 3645-3654 (2005).

62. Heisenberg, M., Wolf, R. \& Brembs, B. Flexibility in a single behavioral variable of Drosophila. Learn. Mem. 8, 1-10 (2001).

63. Wolf, R. \& Heisenberg, M. Basic organization of operant behavior as revealed in Drosophila flight orientation. J. Comp. Physiol. A 169, 699-705 (1991).

64. Kain, J. et al. Leg-tracking and automated behavioural classification in Drosophila. Nat. Commun. 4, 1910 (2013).

65. Kohatsu, S. \& Yamamoto, D. Visually induced initiation of Drosophila innate courtship-like following pursuit is mediated by central excitatory state. Nat. Commun. 6, 6457 (2015).

66. Haberkern, H. et al. Visually guided behavior and optogenetically induced learning in head-fixed flies exploring a virtual landscape. Curr. Biol. 29, 1647-1659.e8 (2019).

67. Erich, B. Elementary movement detectors in an insect visual system. Biol. Cybern. 24, 85-101 (1976).

68. Sun, Y. et al. Neural signatures of dynamic stimulus selection in Drosophila. Nat. Neurosci. 20, 1104-1113 (2017).

69. Warren, T. L., Weir, P. T. \& Dickinson, M. H. Flying Drosophila melanogaster maintain arbitrary but stable headings relative to the angle of polarized light. J. Exp. Biol. 221, jeb177550 (2018).

70. Siju, K. P. et al. Valence and state-dependent population coding in dopaminergic neurons in the fly mushroom body. Curr. Biol. 30, 2104-2115.e4 (2020).

71. Riemensperger, T., Völler, T., Stock, P., Buchner, E. \& Fiala, A. Punishment prediction by dopaminergic neurons in Drosophila. Curr. Biol. 15, 1953-1960 (2005).

72. van Alphen, B., Semenza, E. R., Yap, M., van Swinderen, B. \& Allada, R. A deep sleep stage in Drosophila with a functional role in waste clearance. Sci. Adv. 7, eabc2999 (2021).

73. Sareen, P., Wolf, R. \& Heisenberg, M. Attracting the attention of a fly. Proc. Natl. Acad. Sci. USA 108, 7230-7235 (2011). 
74. Chouhan, N. S., Wolf, R. \& Heisenberg, M. Starvation promotes odor/feeding-time associations in flies. Learn. Mem. 24, 318-321 (2017).

75. Neuser, K., Triphan, T., Mronz, M., Poeck, B. \& Strauss, R. Analysis of a spatial orientation memory in Drosophila. Nature 453, 1244-1247 (2008).

76. Yang, Z., Bertolucci, F., Wolf, R. \& Heisenberg, M. Flies cope with uncontrollable stress by learned helplessness. Curr. Biol. 23, 799-803 (2013).

77. Batsching, S., Wolf, R. \& Heisenberg, M. Inescapable stress changes walking behavior in flies: Learned helplessness revisited. PLoS ONE 11, e0167066 (2016).

78. Götz, K. G. Visual guidance in Drosophila. Basic Life Sci. 16, 391-407 (1980).

79. Baggett, V. et al. Place learning overrides innate behaviors in Drosophila. Learn. Mem. 25, 122-128 (2018).

80. Malisch, J. L. et al. Acute restraint stress alters wheel-running behavior immediately following stress and up to 20 hours later in house mice. Physiol. Biochem. Zool. PBZ 89, 546-552 (2016).

81. Shoji, H. \& Miyakawa, T. Differential effects of stress exposure via two types of restraint apparatuses on behavior and plasma corticosterone level in inbred male BALB/cAJcl mice. Neuropsychopharmacol. Rep. 40, 73-84 (2020).

82. Buynitsky, T. \& Mostofsky, D. I. Restraint stress in biobehavioral research: Recent developments. Neurosci. Biobehav. Rev. 33, 1089-1098 (2009).

83. Gameiro, G. H. et al. Nociception- and anxiety-like behavior in rats submitted to different periods of restraint stress. Physiol. Behav. 87, 643-649 (2006).

84. Marcinkiewcz, C. A. et al. Serotonin engages an anxiety and fear-promoting circuit in the extended amygdala. Nature 537, 97-101 (2016).

85. Xiang, M. et al. Stimulation of anxiety-like behavior via ERK pathway by competitive serotonin receptors $2 \mathrm{~A}$ and $1 \mathrm{~A}$ in posttraumatic stress disordered mice. Neurosignals 25, 39-53 (2017).

86. Fox, E., Ridgewell, A. \& Ashwin, C. Looking on the bright side: Biased attention and the human serotonin transporter gene. Proc. R. Soc. B Biol. Sci. 276, 1747-1751 (2009).

87. Mohammad, F. et al. Ancient anxiety pathways influence drosophila defense behaviors. Curr. Biol. 26, 981-986 (2016).

88. Ries, A.-S., Hermanns, T., Poeck, B. \& Strauss, R. Serotonin modulates a depression-like state in Drosophila responsive to lithium treatment. Nat. Commun. 8, 15738 (2017).

89. Yang, Z. et al. Octopamine mediates starvation-induced hyperactivity in adult Drosophila. Proc. Natl. Acad. Sci. USA 112, 5219$5224(2015)$

90. Gruntenko, N. E. \& Rauschenbach, I. Y. The role of insulin signalling in the endocrine stress response in Drosophila melanogaster: A mini-review. Gen. Comp. Endocrinol. 258, 134-139 (2018).

\section{Acknowledgements}

This work was initiated by observations made by YongJun (Eric) Bae. The author would like to thank Dr. Laura Musselman and Dr. Bryon Tuthill for their help with colorimetric metabolic assay protocols and Dr. Thomas Powell for his help with respirometry preparations and data collection. This work was supported by the Undergraduate Research Award to JG, and by Binghamton university start-up fund and by the National Science Foundation IOS 1457062 award to PM.

\section{Author contributions}

JG and PM designed and performed the experiments, processed and plotted the data, performed the statistics, and wrote the manuscript.

\section{Competing interests}

The authors declare no competing interests.

\section{Additional information}

Supplementary Information The online version contains supplementary material available at https://doi.org/ 10.1038/s41598-021-03575-3.

Correspondence and requests for materials should be addressed to P.M.

Reprints and permissions information is available at www.nature.com/reprints.

Publisher's note Springer Nature remains neutral with regard to jurisdictional claims in published maps and institutional affiliations.

(c) (i) Open Access This article is licensed under a Creative Commons Attribution 4.0 International License, which permits use, sharing, adaptation, distribution and reproduction in any medium or format, as long as you give appropriate credit to the original author(s) and the source, provide a link to the Creative Commons licence, and indicate if changes were made. The images or other third party material in this article are included in the article's Creative Commons licence, unless indicated otherwise in a credit line to the material. If material is not included in the article's Creative Commons licence and your intended use is not permitted by statutory regulation or exceeds the permitted use, you will need to obtain permission directly from the copyright holder. To view a copy of this licence, visit http://creativecommons.org/licenses/by/4.0/.

(C) The Author(s) 2021 